\title{
Optimization and Energy Efficient Analysis of Shortest Path Algorithm in WSN for Node Failure
}

\author{
Preeti Rathore \\ Department of ECE \\ PSIT, Kanpur, Uttar Pradesh, India
}

\author{
Vivek Kumar \\ Department of ECE \\ PSIT, Kanpur, Uttar Pradesh, India
}

\begin{abstract}
The performance of the heterogeneous protocols in terms of stability and network lifetime, DEEC preformed better as compare to others protocols. It is compare the different levels of DEECs performance in terms of number of node alive, number of node fail, stability, network lifetime and energy. Nodes are randomly deployed and each node has initially limited energy. Sensor nodes transmit sensed information to the sink or Base Station (BS) with minimum time delay. When the large numbers of rounds $\left(R_{\max }\right)$ have been involved in the system, the energy has been sharply decreases, so the first node has been died due to low battery and the connection has been broken. Thus, result in unsuccessful information transmission. To overcome this problem, the simulation results of the heterogeneous protocols performance in term of network lifetime, number of nodes alive during rounds and data packets sent to BS.
\end{abstract}

\section{Keywords}

Energy Efficient, heterogeneous protocols life time, stability, node failure, static nodes, mobiles nodes, shortest distance;

\section{INTRODUCTION}

WSN contains numerous sensors nodes having limited power resource, which report sensed data to the base station (BS) that required high energy usage. WSN is most widely used network. In some specific application, continuous data monitoring is prime concern, in such scenario, a significant data loss might be happened due to the node failure problem. As compare to both networks heterogeneous is better perform in different networks. Many algorithms are recently proposed to increase stability and lifetime of heterogeneous WSNs. However, heterogeneous networks are of different types having different Parameters. Every algorithm does not work efficiently for Different networks having different heterogeneity levels and Fails to maintain the same stability period and lifetime as in previous heterogeneous WSNs.

Some algorithms work efficiently in heterogeneous WSNs containing low energy Difference between normal, advanced and super nodes and some algorithms work efficiently in networks containing high Energy difference between normal, advanced and super nodes. So doing interprets in each algorithm of this work, on basis of types of heterogeneous networks containing different heterogeneity Level and parameters on basis of stability period, lifetime of Network and packets sent to the BS. The Heterogeneous protocols SEP, EESAA, and DEEC performance in terms of stability and network lifetime.AS compare to DEEC is better perform than others protocols. This DEEC protocol used to remove the above problem.

\section{WORK DONE}

Wireless Sensor Network (WSN) is the group of tiny sensor nodes having restricted resources of perform given task. Nodes are spotted indiscriminately in the sensing field. In the field coverage and connectivity play an important role in the performance of WSN and in term of static nodes, large number of nodes is required for full coverage and connectivity $[1,2]$. In randomly deployed area predicting the probability of having coverage holes is the most important. Solutions of this problem are either by using many nodes or using mobile nodes in deployed [3]. Increase in static nodes quantity has many disadvantages, like increase in cost of network setup, complex network management, increase in overall power consumption etc. Mobile nodes are movement capable i.e. they can be moved over certain required distance in desired direction. Employing mobile nodes for coverage can reduce number of nodes required to achieve full coverage. In sensor network power saving is another important issue to be considered, as the node life is directly proportion to its battery life. For mobility sensors needed lot of power to move to the desired location. Thus by minimizing the total distance moved by all the mobile nodes, has a direct impact on the power utility on the nodes, thereby maximizes the life of the network, and leads to minimal coverage and connectivity holes [4]. To solving the nodes failure problem due to low battery, using Dijkastra Algorithm And Closest Adjacency Condition (DA-CAC) algorithm involves lest number of nodes during transmission and keeps single nodes active in sleep mode base on Dijkastra algorithm having energy efficiency, at initial level of comparison with exiting mechanism dealing with shortest path suggested approach is proving its presence strongly and will hit the application [8].

The performances of the clustering algorithm on the basis of stability period, network life time and throughput of heterogeneous WSNs its perform under three label energy label difference between normal advance and super nodes in terms of stability period with comparison factor [10]. Hence prolongs the lifetime in WSN has planned and implemented. Proposed approach increase network over all energy, save energy, lifetime and higher performance than LEACH, PEGASIS, TEEN, SEP, DEEC, HEED and EEICCP compare to $\mathrm{LEACH}$, drawback is that when all cluster head dies the network become stable (dead) therefore all the sensors nodes are not active in the network area. Since, this utilized due to in the network does not work till the dead of last node [13].

Distributed energy efficient clustering (DEEC) [14], Developed DEEC [15], Enhance DEEC [16] Threshold DEEC [17] are algorithms considered for heterogeneous WSN. And SEP is designed for two level heterogeneous networks so it cannot work capable in three or multilevel heterogeneous networks. SEP considers only two nodes normal and advanced nodes where the normal nodes consists the low 
energy and advance nodes consists high energy. The DEEC, DDEEC, EDEEC and TDEEC are intended for multilevel heterogeneous networks and can also execute well in two level heterogeneous scenarios. Therefore in this paper discuss and compare the different performance levels of the distributed energy efficient clustered.

\section{RADIO COMMUNICATION MODEL}

Radio Energy Dissipation Model is same as the Radio Energy Model used. Energy model for the radio hardware energy dissipation where the transmitter dissipates energy to run the radio electronics and the power amplifier, and the receiver dissipates energy to sprint the radio electronics. In this model, both are the free space ( $d$ power loss) and the multipath fading ( $d$ power loss) channel models were used in the network, depending on the distance between the transmitter and receiver. Power control can be used to reverse this loss by appropriately location of the power amplifier. If the distance is less than a threshold $d_{0}$, so use the free space model otherwise the multipath model. Thus, transmit an $L$-bit message a distance, the radio expands. The electronics energy $E_{\text {elec }}$ depends on factors as the digital coding, modulation, filtering and distribution of the signal, where as the amplifier energy, $E_{f s} . d_{2}$ or $E_{a m p} . d$ depends on the distance source to the receiver and the acceptable bit-error rate $[2,3]$. In the radio energy model when 1 bit message is transmitted over a distance $d$ as in equation and energy exhausted is then given by:

$$
E_{T x}(l, d)=\left\{\begin{array}{ll}
l E_{\text {elec }}+l \varepsilon_{f s} d^{2}, & \mathrm{~d}<\mathrm{d}_{\mathrm{o}} \\
l E_{\text {elec }}+l \varepsilon_{m p} d^{4}, & \mathrm{~d} \geq \mathrm{d}_{0}
\end{array}\right\}
$$

Where, $E_{\text {elec }}$ is the energy dissipated per bit to scamper the transmitter or the receiver circuit. And $d$ is the distance between the sender and receiver. If this distance is less than threshold, so used free space $(f s)$ model else multi path ( $m p$ ) model now, total energy dissipated in the network during a round is:

$$
E_{\text {round }}=L\left(2 N E_{\text {elec }}+N E_{D A}+k \varepsilon_{m p} d_{t o B S}^{4}+N \varepsilon_{f s} d_{t o C H}^{2}\right)
$$

Where, $\mathrm{K}=$ number of clusters

$$
E_{D A}=\text { Data aggregation cost expended in } \mathrm{CH}
$$

$d_{\text {to }} B S=$ Average distance between the $\mathrm{CH}$ and BS

$d_{t o} C H=$ Average distance between the cluster members and the $\mathrm{CH}$

$$
\begin{aligned}
d_{t o} C H & =\frac{M}{\sqrt{2 \pi k}} \\
d_{t o} B S & =0.765 \frac{M}{2}
\end{aligned}
$$

And

$$
k_{o p t}=\frac{\sqrt{N}}{\sqrt{2 \pi}} \sqrt{\frac{\varepsilon_{f s}}{\varepsilon_{m p}}} \frac{M}{d_{t o B S}^{2}}
$$

\section{NETWORK MODEL}

Network model is used to formed $N$ number of nodes in $M \times M$ network field. Since, the Sensor Nodes are consistent randomly deployed in the network. Consider the one Base Station which is located at the centre of the sensing field. And nodes are always having the data that is send to the base station. Nodes are location-oblivious that is not equipped with GPS-capable antenna. Hence, all nodes have similar capabilities in terms of processing and communication and of equal significance.

Table 3.1 Parameters used in simulations Parameter Value.

\begin{tabular}{|l|l|}
\hline Parameter & Value \\
\hline$E_{\text {elec }}$ & $5 \mathrm{~nJ} /$ bit $\mathrm{c}$ \\
\hline$\varepsilon_{f}$ & $10 \mathrm{pJ} / \mathrm{bit} / \mathrm{m}^{2}$ \\
\hline$\varepsilon_{m p}$ & $0.0013 \mathrm{pJ} / \mathrm{bit} / \mathrm{m}^{4}$ \\
\hline$E_{0}$ & $0.5 \mathrm{~J}$ \\
\hline$E_{D A}$ & $5 \mathrm{~nJ} /$ bit / message \\
\hline$d_{0}$ & $70 \mathrm{~m}$ \\
\hline Message size & 4000 bits \\
\hline$p_{\text {opt }}$ & 0.1 \\
\hline
\end{tabular}

Where: $E_{\text {elec }}=$ Electronics energy.

$\varepsilon_{f}=$ Free space efficiency.

$\varepsilon_{m p}=$ Multi path efficiency.

$E_{0}=$ initial energy.

$E_{D A}=$ information aggregation cost expended in $\mathrm{CH}$.

$d_{0}=$ Threshold distance.

$p_{\text {opt }}=$ optimal power.

\section{OVERVIEW OF DISTRIBUTED HETEROGONOUS PROTOCOLS}

There are four types of heterogeneous protocols that are: DEEC, DDEEC, EDEEC and TDEEC. DEEC (distributed energy efficient clustering algorithm). DEEC uses the initial and residual energy level of the nodes to select the clusterheads. To avoid that each node needs to know the global knowledge of the networks, DEEC estimates the ideal value of network life-time, which is use to compute the reference energy that each node should expend during a round. To describe DEEC, an energy-aware adaptive clustering protocol used in heterogeneous wireless sensor networks.

In DEEC, every sensor node independently elects itself as a cluster-head based on its initial energy and residual energy. To control the energy expenditure of nodes by means of adaptive approach, DEEC use the average energy of the network as the reference energy. Thus, DEEC does not require any global knowledge of energy at every election round. Unlike SEP and LEACH, DEEC can perform well in multilevel heterogeneous wireless sensor networks. 
Let $n_{i}$ denote the number of rounds to be a $\mathrm{CH}$ for node $s_{i} \cdot p_{\text {opt }} N$ is the optimum number of $\mathrm{CHs}$ in the network during each round. As in homogenous network, when nodes have same amount of energy during each epoch then $p_{i}=p_{\text {opt }}$ assures that $p_{\text {opt }} N \mathrm{CHs}$ during each round. In WSNs, nodes with high energy are more probable to become $\mathrm{CH}$ than nodes with low energy but the net value of $\mathrm{CHs}$ during each round is equal to $p_{o p t} N \cdot P_{i}$ is the probability for each node in the set to become $\mathrm{CH}$, so, node with high energy has larger value of $p_{i}$ as compared to the $p_{\text {opt }} \cdot \bar{E}(r)$ denotes average energy of network during round $r$ so energy:

$$
\bar{E}(r)=\frac{1}{N} \sum_{i=1}^{N} E_{i}(r)
$$

Therefore the Probability for $\mathrm{CH}$ selection in DEEC

$$
\begin{aligned}
p_{i} & =p_{o p t}\left[1-\frac{\bar{E}(r)-E_{i}(r)}{\bar{E}(r)}\right] \\
& =p_{o p t} \frac{E_{i}(r)}{\bar{E}(r)}
\end{aligned}
$$

Now the average total number of $\mathrm{CH}$ during each round is:

$$
\begin{aligned}
\sum_{i=1}^{N} p_{i} & =\sum_{i=1}^{N} p_{o p t} \frac{E_{i}(r)}{\bar{E}(r)} \\
& =p_{o p t} \sum_{i=1}^{N} \frac{E_{i}(r)}{\bar{E}(r)} \\
& =N p_{o p t}
\end{aligned}
$$

Hence, the probability $p_{i}$ is probability of each node to become $\mathrm{CH}$ in a round. And $G$ is the set of nodes eligible to become $\mathrm{CH}$ at round $r$. During each round each node chooses a random number between 0 and 1 . If number is less than threshold as defined in equation (12)

$$
T\left(s_{i}\right)=\left\{\begin{array}{l}
\frac{p_{i}}{1-p_{i}\left(r \bmod \frac{1}{p_{i}}\right)} \text { if } s_{i} \in G \\
0 \quad \text { otherwise }
\end{array}\right\}
$$

As $p_{\text {opt }}$ is reference value of average probability $p_{i}$. In heterogeneous networks, the value of $p_{\text {opt }}$ is different according to the initial energy of the node. In two levels heterogeneous network the value of $p_{o p t}$ is;

$$
\begin{aligned}
p_{a d v} & =\frac{p_{o p t}}{1+a m} \\
p_{n r m} & =\frac{p_{o p t}(1+a)}{1+a m}
\end{aligned}
$$

And average probability is:

$$
p_{i}=\left\{\begin{array}{l}
\frac{p_{\text {opt }} E_{i}(r)}{(1+a m) \bar{E}(r)} \text { if } s_{i} \text { is the normal node } \\
\frac{p_{\text {opt }}(1+a) E_{i}(r)}{(1+a m) \bar{E}(r)} \text { if } s_{i} \text { is the advanced node }
\end{array}\right\}
$$

Therefore the above model can also be extended to multi level heterogeneous network given as:

$$
p_{m u l t i}=\frac{p_{o p t} N\left(1+a_{i}\right)}{\left(N+\sum_{i=1}^{N} a_{i}\right)}
$$

To get $p_{i}$ for heterogeneous node in term of $p_{o p t}$ the multilevel heterogeneous network is:

$$
p_{i}=\frac{p_{o p t} N(1+a) E_{i}(r)}{\left(N+\sum_{i=1}^{N} a_{i}\right) \bar{E}(r)}
$$

In DEEC the estimate average energy $E(r)$ of the network for any round $r$ as:

$$
\bar{E}(r)=\frac{1}{N} E_{\text {total }}\left(1-\frac{r}{R}\right)
$$

Here, $\mathrm{R}$ denotes total rounds of network lifetime and is estimated as follows:

$$
\boldsymbol{R}=\frac{E_{\text {total }}}{E_{\text {round }}}
$$

Since, $E_{\text {total }}$ is total energy of the network and $E_{\text {round }}$ is energy expenditure during each round.

\section{D-DEEC (Developed Distributed Energy-Efficient Clustering):}

Difference between DDEEC and DEEC is centered in expression that defines probability for normal and advanced nodes to be a $\mathrm{CH}$ [14] as given in equation (12). A point comes in a network where advanced nodes having same residual energy like normal nodes. Although, after this point DEEC continues to punish the advanced nodes so this is not optimal way for energy distribution because by doing so, advanced nodes are continuously a $\mathrm{CH}$ and they die more quickly than normal nodes. To avoid this unbalanced case, DDEEC makes some changes in equation (12) to save advanced nodes from being punished over and again. DEEC introduces threshold residual energy as in [15] and given below:

$$
T h_{R E V}=E_{0}\left(1+\frac{a E_{d i s N N}}{E_{d i s N N}-E_{d i s A N}}\right)
$$

When energy level of advanced and normal nodes falls down to the limit of threshold residual energy then both type of nodes use same probability to become cluster head. Therefore, $\mathrm{CH}$ selection is balanced and more efficient. Threshold residual energy $T h$ is:

$T h_{\text {REV }} \square(7 / 10) E_{o}$ 
Average probability $p_{i}$ for $\mathrm{CH}$ selection used in DDEEC is:

$$
p_{i}=\left\{\begin{array}{l}
\frac{p_{\text {opt }} E_{i}(r)}{(1+a m) \bar{E}(r)} \text { for Nml nodes, } E_{i}(r)>T h_{R E V} \\
\frac{(1+a) p_{\text {opt }} E_{i}(r)}{(1+a m) \bar{E}(r)} \text { for Adv nodes, } E_{i}(r)>T h_{R E V} \\
c \frac{(1+a) p_{o p t} E_{i}(r)}{(1+a m)^{-} \bar{E}(r)} \text { for Adv, Nml nodes, } E_{i}(r) \leq T h_{R E V}
\end{array}\right\}
$$

\section{E-DEEC (Enhanced Distributed Energy Efficient Clustering):}

EDEEC protocols design based on DEEC with addition of super nodes. Since, extended the DEEC to three levels heterogeneous in the network model described in previous section some assumptions have been made for the sensor nodes as well as for the network. Hence the assumptions and properties of the network and sensor nodes are;

- Sensor Nodes are uniformly randomly deployed in the network.

- There is only a Base Station which is located at the centre of the sensing field.

- Nodes always have the data to send to the base station.

- The location of Nodes is unaware, i.e. not equipped with GPS capable antennae.

- All nodes have similar capabilities in terms of processing, communication and equal significance.

This motivates needs for extending the lifetime of every sensor. Sensor nodes have heterogeneity in terms of energy i.e. different energy levels. So, all the nodes have different initial energy; some nodes are equipped with more energy than the normal nodes [16]. Therefore it contains three types of nodes normal, advanced and super nodes based on initial energy. Here $p_{i}$ is probability used for $\mathrm{CH}$ selection and $p_{o p t}$ is reference for $p_{i}$ and EDEEC uses different $p_{o p t}$ values for normal, advanced and super nodes, so, value of $p_{i}$ in EDEEC is:

$$
p_{i}=\left\{\begin{array}{l}
\frac{p_{\text {opt }} E_{i}(r)}{\left(1+m\left(a+m_{o} b\right)\right) \bar{E}(r)} \text { if } s_{i} \text { is the normal node } \\
\frac{p_{o p t}(1+a) E_{i}(r)}{\left(1+m\left(a+m_{o} b\right)\right) \bar{E}(r)} \text { if } s_{i} \text { is the advanced node } \\
\frac{p_{\text {opt }}(1+b) E_{i}(r)}{\left(1+m\left(a+m_{o} b\right)\right) \bar{E}(r)} \text { if } s_{i} \text { is the super node }
\end{array}\right\}
$$

Threshold for $\mathrm{CH}$ selection for all three types of node is:

$$
T(s i)=\left\{\begin{array}{l}
\frac{p_{i}}{1-p_{i}\left(\operatorname{rmod} \frac{1}{p_{i}}\right)} \text { if } p_{i} \in G^{\prime} \\
\frac{p_{i}}{1-p_{i}\left(\operatorname{rmod} \frac{1}{p_{i}}\right)} \text { if } p_{i} \in G^{\prime \prime} \\
\frac{p_{i}}{1-p_{i}\left(\operatorname{rmod} \frac{1}{p_{i}}\right)} \text { if } p_{i} \in G^{\prime \prime \prime} \\
0 \quad \text { otherwise }
\end{array}\right\}
$$

\section{T-DEEC (Threshold Distributed Energy Efficient Clustering):}

In cluster based DEEC algorithm in which cluster heads are selected on the basis of probability of ratio of residual energy and average energy of the network. In this algorithm, node having more energy has more chances to be a cluster head. It prolongs the lifetime of the network. TDEEC (Threshold Distributed Energy Efficient Clustering) scheme follows all the thoughts of DEEC. This scheme selects the cluster heads from the high energy nodes improving energy efficiency and lifetime of the network. TDEEC uses same mechanism for $\mathrm{CH}$ selection and average energy estimation as proposed in DEEC. At each round, nodes decide whether to become a $\mathrm{CH}$ or not by choosing a random number between 0 and 1 . If number is less than threshold as shown in equation (21) then nodes decide to become a $\mathrm{CH}$ for the given round. In TDEEC, threshold value is adjusted and based upon that value a node decides whether to become a $\mathrm{CH}$ or not by introducing residual energy and average energy of that round with respect to optimum number of CHs [17]. Threshold value proposed by TDEEC is:

$$
T(s)=\left\{\frac{p}{1-p\left(\operatorname{rmod} \frac{1}{p}\right)} \frac{\text { residual energy of a node } \times k_{\text {opt }}}{\text { average energy of the network }}\right\}
$$

One Base Station which is located at the centre of the sensing field. And nodes are always having the data that is send to the base station. Nodes are location-oblivious that is not equipped with GPS-capable antenna. Hence, all nodes have similar capabilities in terms of processing and communication and of equal significance.

\section{ALGORITHM OF DEEC}

Now represent the algorithm of distributed energy efficient clustering protocols.

- $\quad$ First initialized the general parameters and formula of Radio model networks in the fixed area which has a dimension of $x$ axis and $y$ axis is 100 meters and randomly initialized the 100 number of nodes or more then 100 nodes.

- Here the protocols are passing through the three nodes normal, advance and super. And they represent as for normal nodes $n *(1-m)$, for advance $\operatorname{nodes} n * m *\left(1-m_{0}\right)$ and for super nodes $n * m * m_{0}$.

- Initially the value of $m=0.5, m_{0}=0.4$, the fraction of energy enhancement of advanced nodes at $a=1.5$ and the fraction of extra energy in super nodes at $b=3$.

- Choose the various nodes $n_{i}$ based on residual energy $E_{i}(r)$ of source node $S_{i}$ at round $r$.

- Initially there are no cluster heads when nodes are obtainable in the networks. Compute the separation between cluster head and base station by $d_{1}=0.765 * x_{m} / 2$, optimal number of cluster heads 
distance between cluster members and cluster heads through another distance formula $d_{2}=x_{m} / \operatorname{sqrt}(2 * \mathrm{pi} * K)$ and the energy degenerate in a round by formula

$E_{r}=4000\left(2 * n * E_{T X}+n * E_{D A}+K * E_{m p} * d_{1}^{4}+n * E_{f_{s}} * d_{2}^{2}\right)$

- Initially $r=0$ the power exponent $n$ and the time of the rounds are predefine and also assume there are zero nodes dead initially.

- Node $i$ sends its initial energy to the base station. According to the receiving message of all nodes so the base station determines the various parameters that are; $N, E_{\text {total }}, R, E_{\max }$ and $E_{\min }$ in the whole predefine networks.

- Determine the shortest distance between the nodes. If cluster head is there survive then choose if. Further optimized the weight value by the shortest path algorithm.

- Select the node by the optimized weight value then that node works as the cluster head. Every cluster head broadcast a message to announce itself as a cluster head and the non cluster head joints the nearest cluster head.

- The cluster head assigns a transmission slot to each of its cluster member and these members transmit the sensing data to the cluster head in the designated slot or sink.

- The end of current nodes by $r=r+1$. Now back to step 8.

- Otherwise end the process and shoes the output in term of node dead, node alive, packet sent to the base station and number of cluster heads.

\section{SIMULATION}

Now the performances of heterogeneous protocols in WSNs are shown below at different stages. Here combined all four algorithm and compare to the each other at three different stages at different point's first nodes dead second number of nodes alive and third packets sent to the base station. And the points are containing $m=0.5$ fraction of advanced nodes having $a=1.5$ times more energy and $m o=0.4$ fraction of super nodes containing $b=3$ times more energy than normal nodes at the first node for DEEC, DDEEC, EDEEC and TDEEC dies at $1039,1274,1433$ and 1433 rounds respectively. Tenth node dies at 1476, 1626, 1608 and 1608 rounds respectively.

All nodes are dead at 3955, 3541, 0 and 0 rounds respectively. It is obvious from the results of all protocols that in terms of stability period, TDEEC and EDEEC perform best of all; EDEEC performs better than DEEC and DDEEC but has less performance than TDEEC and EDEEC. DDEEC only performs well as compared to DEEC and DEEC has least performance than all the protocols. Stability period of DEEC and DDEEC is lower than EDEEC and TDEEC because the probabilities in TDEEC and EDEEC are defined separately for normal, advanced and super nodes whereas, DEEC and DDEEC do not use different probabilities for normal, advanced and super nodes so their performance is lower than EDEEC and TDEEC.

However, instability period of EDEEC and TDEEC is much larger than DEEC and DDEEC. The number of nodes alive in TDEEC is quite larger than EDEEC because in TDEEC the formula of threshold used by nodes for $\mathrm{CH}$ election is modified by including residual and average energy of that round. So nodes having high energy will become CHs. Similarly, by examining results, packets sent to the BS by DEEC, DDEEC, EDEEC and TDEEC have their values at 95831, 83376, 332778 and 332874.

Now this shows the packets sent to BS for DEEC and DDEEC is almost same whereas, the packets sent to BS for EDEEC and TDEEC are almost the same because the probability equations for normal, advanced and super nodes is same in both of them. Now coming to the $\mathrm{CHs}$, the packets sent to $\mathrm{CHs}$ increase during the start of the network and gradually decrease down towards the end due to the nodes dying simultaneously.

Now considering further case, parameter values further decrease to $a=1.1, b=2, m=0.3, m o=0.2$ in which first node for DEEC, DDEEC, EDEEC and TDEEC dies at 1153, 1335, 1376, and 1376 rounds respectively. Tenth node dies at 1394, 1496, 1654 and 1660 rounds respectively.

All nodes are dead at 3135, 2935, 0 and 0 rounds respectively. Similarly, the packets to BS sent in DEEC, DDEEC, EDEEC and TDEEC are 76382, 77877, 259160 and 259165 respectively. Now considering fourth case, parameters are increased to $a=1.7, b=3.5, m=0.6, m o=0.5$. Results show that Packets sent to the BS in DEEC, DDEEC, EDEEC and TDEEC are 107101, 83709, 365599 and 365599. Now in last case considering multilevel heterogeneous net-work here see that for DEEC, DDEEC, EDEEC and TDEEC first node dies at $1275,1188,1364$ and 1366 rounds respectively.

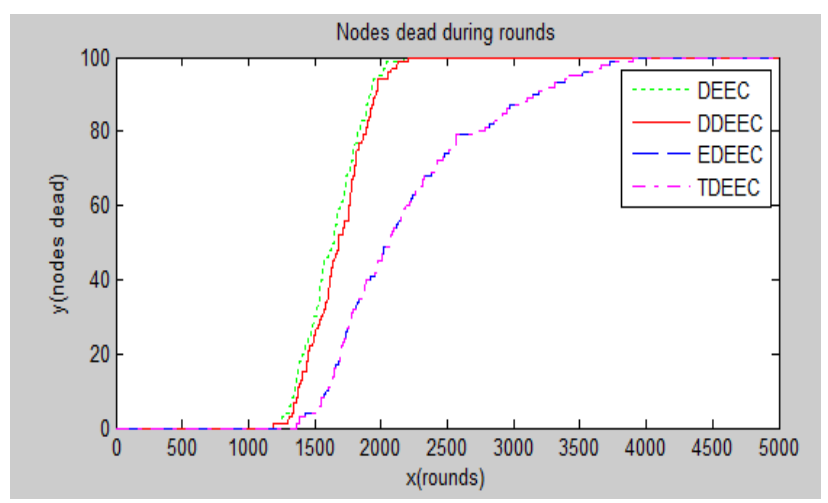

Figure 1: Nodes dead during the rounds.

Tenth node dies at 1345, 1374, 1587, 1588 rounds respectively and all nodes are dead at 2175, 2207, 3904 and 3906 rounds shows in figure 1and figure 2 shows the number of node alive during the round.

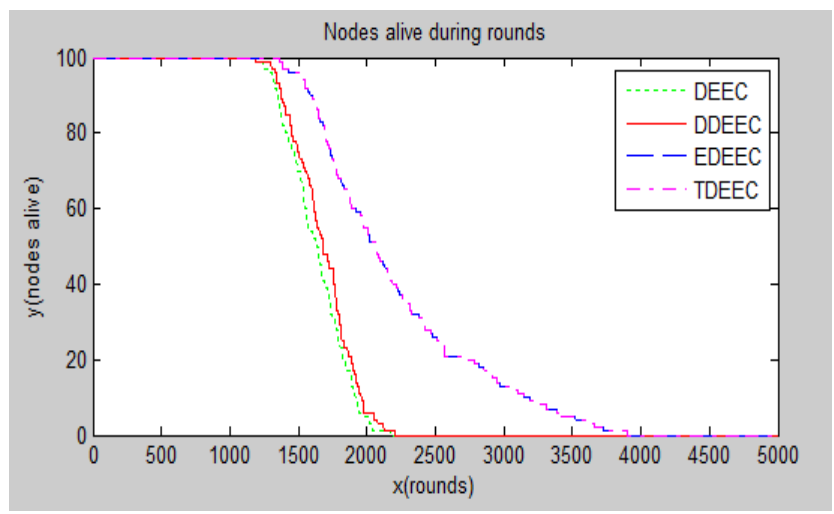

Figure 2: Nodes alive during the rounds. 
Packets sent to the BS in DEEC, DDEEC, EDEEC and TDEEC are 48412, 60930, 220902 and 220904 respectively shown in figure 3 .

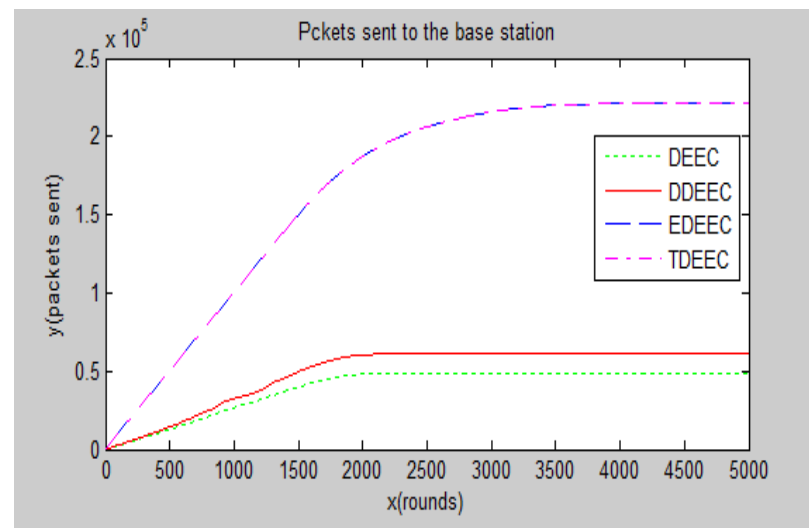

Figure 3: Packets sent to the base station.

It is observed from all the above scenarios that for first case of three level heterogeneous WSN. Considering $\mathrm{a}=1.5, \mathrm{~b}=3, \mathrm{~m}=$ 0.5 and mo $=0.4$ TDEEC performs best of all, EDEEC performs better than DDEEC and DEEC where DDEEC performs better than DEEC in terms of stability period. For EDEEC and TDEEC instability period is higher as compared to DDEEC and DEEC.

When values of $a, b, m, m o$ are decreased linearly further in second and third scenario, same results as in first scenario are found for all protocols. In fourth and fifth scenarios when $a, b$, $m, m o$ are increased linearly it is found after larger number of simulations that in some scenarios DEEC performs better than DDEEC, EDEEC in terms of stability period, TDEEC performs best and stability period of DDEEC and EDEEC is almost the same. Whereas instability period of TDEEC and EDEEC is also larger than DEEC and DDEEC even some nodes are not dead in EDEEC and TDEEC after 5,000 rounds. In last case considering multilevel heterogeneous network in which all nodes have random energy it is observed that TDEEC performs best of all, EDEEC performs better than DDEEC and DEEC and DDEEC performs better than DEEC in terms of stability period. For EDEEC and TDEEC instability period is higher as compared to DDEEC.

\section{CONCLUSION}

In this paper discuss the node failure problem in the wireless sensors networks when the large numbers of nodes are activated in the network for a long time so some of the nodes have loosen its own initial energy at that time that nodes comes into the failure modes to reduce this problem used the heterogeneous protocols in the networks and study the behavior of these protocols since, the result of DEEC, EDEEC, T-DEEC and D-DEEC protocols for heterogeneous WSNs containing different level of heterogeneity. Simulations of this paper have to show that the performance of the parameters of DDEEC performed better as compare to DEEC parameters in same values and the networks containing high energy difference between normal, advanced and super nodes. And also the EDEEC protocols perform well in the same parameters and TDEEC protocols perform best as compare to all previous three protocols in all values. TDEEC protocol has a best performance in terms of stability period and existence time but instability period of EDEEC and TDEEC is very large. Since, EDEEC and TDEEC are improved in terms of stability period while compromising on lifetime therefore when it's taken around 5000 rounds so the output is become stable.

\section{FUTURE SCOPE}

In future the same problem will be generating again so compute the algorithm for a more rounds above 8000 rounds the instability are create that is the issue in lifetime between Enhance and threshold DEECs protocols because these two protocols are perform better than DEEC and Developed DEEC protocols. If any case the node failure problem is generating in the network. After checking the performance of super stable nodes case, the super stable nodes are exists in the Enhance and threshold protocols and the performance of these heterogeneous protocols which further research can be done on the above mentioned issue.

\section{ACKNOWLEDGMENTS}

I am very grateful to my supervisor, "Dr. Vivek Kumar", Lecturer, Department of Electronic and Communication Engineering. Whose tolerant encourages supervision and insightful criticism from the beginning to the final level facilitate me have a deep accepting of the thesis.

I am highly grateful to "Dr. Ram Chandra Singh Chauhan", Head, Department of Electronics and communication Engineering, Pranveer Singh Institute of Technology for providing necessary facilities and encouraging his sincere thanks to all the faculty members of the Department of Electronics and Communication Engineering for their help and encouragement.

I would also like to thank all my faculty and staff of Electronics and Communication Engineering Department, Pranveer Singh Institute of Technology, Kanpur for their support and encouragement during the two years of my student life in the institution.

I offer my insightful observe and blessing to everyone those are supported me in any conditions during the completed of my thesis. I also thank my parents for their unceasing encouragement and support and friends in every way offered much assistance before during and at competitions stage of this thesis work.

\section{REFERENCES}

[1] Guo-Lie Lan, Zhi-Ming $\mathrm{Ma}$ and Su-Yong Sun ,"Coverage Of Wireless Sensor Networks.",2002

[2] S.K L V Sai Prakash and P. Niranjan." Area Based Covered For Three Deployment Distributions And Their Connectivity In Wireless Sensor Networks." The International Conference on Communication Systems and Network Technologies, 2014.

[3] E.P.De Freitas T. Heimfarth, I.F Netto, C.E. Pereira, A.M. Ferreira F.R. Wagner, and T. Larsson, "Handling Failure of Static Sensor Nodes in Wireless Sensor Network by Use of Mobile Sensors." In Advanced Information Networking and Applications (Waina), 2011 IEEE Workshops of International Conference On. IEEE, 2011, Pp.127-134.

[4] S.K L V Sai Prakash and P. Niranjan," Movement Minimization of Randomly Deployed Mobile Nodes for Complete Coverage and Connectivity." The International Conference on Advance Communication Control and Computing Technologies (ICACCCT), 2014.

[5] Seema V, Pinki, N, And Rekha Agarwal, "Energy Efficient Routing In Mobile Adhoc Networks Based On AODV Protocol," International Journal Of Computer Science. Nov, 2012. 
[6] Sourabh Jain, Praveen Kaushik, and Jyoti Singhai, "Energy Efficient Maximum Lifetime Routing For Wireless Sensor Network." International Journal Of Advanced Smart Sensor Network Systems (IJSSAN); Jan 2012.

[7] Sarvesh Singh Rai, D. Ahirwar," Improvement Of AODV Routing Protocol Algorithm With Link Stability And Energy Efficient Routing For MANET , International Journal Of Computer Science Engineering And Technology(IJCSET) Feb 2014.

[8] Neeti Nema," Gateway Based Shortest Path Detection Using Dijkastra's Algorithm and Closest Adjacency Condition (DA-CAC) In WSN." International Journal of Advanced Research in Computer Science and Software Engineering. Nov 2014

[9] Jin W, J.U.Kim, L. Shu, Yu Niu and S. Lee," A Distance Based Energy Aware Routing Algorithm for WSN."SENSORS 2010.

[10] P. Rani, Satish Kumar," New Cluster Making Scenario for Next Generation System Using Graph Theory System.” IJRECE, Apr- June 2015.

[11] Jin Wang, Z. Zhang, F. Xia, W. Yuan and S. Lee, “An Energy Efficient Stable Election Based Routing Algorithm for Wireless Sensor Networks," Sensors 2013.

[12] M. Kamath, Nandini .M," Cluster Head Selection Algorithm to Minimize Energy Consumption in WSN,'IJIRCCE May 2014, ICACCE (ACCE- Apr 2014).

[13] Monika Raghatate And Prof Dipak W. Wajgi," An Energy Saving Algorithm To Prolong The Lifetime Of WSN. International Journal of Wireless and Mobile Network (IJWMN), Oct 2014.

[14] L.Qing, Q. Zhu, M. Wang," Design Of A Distributed Energy Efficient Clustering (DEEC) Algorithm for Heterogeneous Wireless Sensor Networks," Science Direct, Elsevier 10 March 2006.
[15] Bra him E., S.Rachid, Sanna E .L.Fkihi, Driss, "A Developed Distributed Energy Efficient Clustering (DDEEC) For Heterogeneous WSNs," IEEE 2010.

[16] Parul Saini, Ajay .K. Sharma," Enhanced Distributed Energy Efficient Clustering (EDEEC) Scheme for Heterogeneous WSN. 2010 1st International Conference on Parallel, Distributed and Grid Computing.

[17] Parul Saini, Ajay .K. Sharma,"Energy Efficient Scheme for Clustering Protocol Prolonging the Lifetime of Heterogeneous WSN (TDEEC).”International Journal Of Computer Application Sep

[18] Georgiou S. Ibrahim Matta, Azer. B "Sep- A Stable Election Protocol for Clustering Heterogeneous Wireless Sensor Networks."

\section{AUTHOR PROFILE}

Preeti Rathore was born in Kanpur,UP, India, in 1989. She recieved Bachelor of Technology degree in Electronics \& Communicat ion Engineering from UPTU, Lucknow, India. She joined M.Tech in Electronics \& Communication Engineering from Dr. A. P. J. Abdul Kalam Technical Un iversity, Lucknow, India in 2013.

Vivek Kumar was born in Ghazipur, india, in 1988. He received Bachelor of Technology degree in Electronic \& Communication Engineering from UPTU, Lucknow, India and M.tech degree in Electronic and communication Engineering from Jaypee Institute of Information Technology University, Noida, India, in 2009 and 2011 respectively. From January 2012 to September 2012, he was an Assistant Professor in DITS, Allahabad, India. Since, October 2012, he has been working as a Research Scholar (Ph.D.) in the department of Electronics and Co mmunication Engineering at Birla Institute of Tech-nology Mesra, Ranchi, India. His current research fields are Wireless Body Area Network, electrically small antennas, UWB Body-wo rn antenna, and MAC layer issue in WBAN. 